University of Texas at El Paso

ScholarWorks@UTEP

$2-2003$

\title{
Detection of Cracks at Rivet Holes in Thin Plates Using Lamb- Wave Scanning
}

Roberto A. Osegueda

The University of Texas at El Paso, osegueda@utep.edu

Vladik Kreinovich

The University of Texas at El Paso, vladik@utep.edu

Soheil Nazarian

The University of Texas at El Paso, nazarian@utep.edu

Enrique Roldan

Follow this and additional works at: https://scholarworks.utep.edu/cs_techrep

Part of the Computer Engineering Commons

Comments:

Technical Report: UTEP-CS-03-09

Published in: Tribikram Kundu (ed.), Smart Nondestructive Evaluation and Health Monitoring of Structural and Biological Systems II, Proceedings of the SPIE/International Society for Optical Engineering, Vol. 5047, San Diego, CA, March 3-5, 2003, pp. 55-66.

\section{Recommended Citation}

Osegueda, Roberto A.; Kreinovich, Vladik; Nazarian, Soheil; and Roldan, Enrique, "Detection of Cracks at Rivet Holes in Thin Plates Using Lamb-Wave Scanning" (2003). Departmental Technical Reports (CS). 283.

https://scholarworks.utep.edu/cs_techrep/283

This Article is brought to you for free and open access by the Computer Science at ScholarWorks@UTEP. It has been accepted for inclusion in Departmental Technical Reports (CS) by an authorized administrator of ScholarWorks@UTEP. For more information, please contact Iweber@utep.edu. 


\title{
Detection of cracks at rivet holes in thin plates using Lamb-wave scanning
}

\author{
Roberto Osegueda, Vladik Kreinovich, Soheil Nazarian and Enrique Roldan, \\ FAST Center for Structural Integrity of Aerospace Systems \\ The University of Texas at El Paso \\ El Paso, Texas 79968 \\ Contact Email Address: osegueda@utep.edu
}

\begin{abstract}
This paper describes a Lamb-wave scanning method for the detection of notches simulating cracks at rivet holes in thin plates. The approach requires the generation of an ultrasonic $\mathrm{S}_{0}$-Mode Lamb wave using an incident transmitter excited with a tone burst centered at a near non-dispersive frequency. Area scans are performed on a plate with a hole with a notch to generate times series information which is used to create animations illustrating the wave propagation characteristics. The time series are subject to a sifting process to obtain intrinsic mode functions which contain narrow frequency banded information of the signals. The Hilbert-Huang transform is applied to the intrinsic mode functions which permit the computation of the signal energy as a function of time, proportional to the square of the amplitude of the analytical signal. Animations of the propagation of the Lamb-wave energy illustrate that a potential scanning approach is to acquire time series along a line between the transmitter and the hole, capturing wave scattering from the hole and reflections from the notches. The times of flight and amplitudes of the notch-reflected energy are used to calculate coordinates of the source of the reflections by a geometric approach. The identified coordinates of the reflections outline the extent of the notch at the rivet hole. Results of experiments conducted on thin square plates with a single hole with notches of various sizes compare favorably with the actual notches.
\end{abstract}

Keywords: Lamb waves, ultrasonics, waveforms, nondestructive evaluation, defect localization.

\section{INTRODUCTION}

One of the most common problems in aging aircraft structures is the presence of cracks initiated at rivet holes. The visual inspection for this type of defects is time consuming and impractical. Moreover, the defects might not be visible because they may be hidden inside the structure or covered with paint. Although, their detection with Eddy current and ultrasonic techniques is already well established, the use of these methods requires the inspection of rivets one at a time. One approach that has the potential for improving the inspections of rivet holes is the use of Lamb waves.

The advantages of using ultrasonic Lamb waves as a method for inspecting large areas have been previously recognized. Cawley and Alleyne [1] realized the attractiveness of using Lamb waves for the inspection of large areas due to their capabilities of propagating long distances. They discussed that the key for the successful application of the method is the proper excitation of a single mode at a frequency located in a near non-dispersive region. Kundu et al. [2] presented a scanning technique for composite materials using leaky Lamb waves using a pitch-catch mode involving an immersed transmitter and a receiver. Their technique was effective in detecting internal defects such as missing fibers and fiber breakage. Lowe et al. [3] studied issues on Lamb wave sensitivity to defects and optimum selection of modes as related to defect size and the strength of wave reflections. Other studies have focused on sensitivity issues to detect certain forms of damage. Ghosh et al. [4] studied Lamb wave propagation in large plates and its use in internal defect detection by computing stress fields inside the plate for different Lamb modes. They inferred which mode should be more efficient for detecting a particular type of material defect. Kundu et al. [5] investigated the effectiveness of using Lamb waves for the detection of kissing bonds, defined as closed cracks under compressive normal stresses. Their technique was based on the proper selection of the $\mathrm{A}_{1}$ mode, which proved sensitive to cause excitations in the interface of the

SPIE USE, V. 3 5047-6 (p.1 of 12) / Color: No / Format: Letter/ AF: Letter / Date: 2003-02-16 22:39:44 
crack's surfaces. Zhu et al. [6] documented an experimental study of hidden corrosion detection using ultrasonic guided waves. Chang and Mal [7] theoretically and experimentally investigated Lamb waves propagating in an infinite plate containing a circular hole with or without edge cracks. The theoretical analysis was accomplished by means of a hybrid method involving finite elements with special boundary condition fields representing Lamb wave modes. Measured time histories and amplitude spectra of the transmitted and reflected waves compared favorably with those calculated from the hybrid model. Chang and Mal [7] also observed that when Lamb waves pass through a region with cracks or material loss, some energy is reflected back due to back scattering and the transmitted waves are modified due to forward scattering. The presence of material loss, cracks or other flaws in the path of the incident Lamb waves can be detected and, in principle, characterized by analyzing the characteristics of the reflected and transmitted waves.

This paper deals with the detection of notches at holes using line scans of ultrasonic Lamb waves propagating in the $\mathrm{S}_{\mathrm{o}}$ mode in thin plates with constant thickness. The approach relies on the generation of narrow-band pulse waves with a center frequency in the non-dispersive region of the dispersion curve. The signals acquired from a wide-band ultrasonic receiver, housed within a bubbler and mounted in a scanning arm, are processed using the Hilbert-Huang transform to determine wave energy as a function of time. The points of maximum energy are used to determine arrival times. From the arrival times acquired at two different receiver positions, the distances traveled from the source to the flaw, and to the receivers serve as the bases for a geometrical solution for the flaw location after identifying a region not affected by the wave back scattering due to the hole geometry.

\section{EXPERIMENTAL SETUP}

A typical specimen considered in this investigation is illustrated in Figure 1. The specimens consisted of 6061-T6 30 $\mathrm{cm}$ square Aluminum plates with a thickness of $1.60 \mathrm{~mm}(1 / 16 \mathrm{in}$.$) and a 1.27-\mathrm{cm}(0.5 \mathrm{in}$.$) center hole. Various plates$ were fabricated with full-thickness notches ranging from $3.2 \mathrm{~mm}$ (1/8 in.) to $25.4 \mathrm{~mm}$ (1 in.) long in $3.2-\mathrm{mm}$ increments. The notches were perpendicular to one of the sides. The ultrasonic Lamb waves were created by sending a signal from a function generator through a continuous amplifier to a transmitter mounted on a Plexiglas wedge. The wedge controlled the incidence angle of the excitations at 31 degrees from an imaginary line normal to the plate. This angle was chosen to optimize the excitation of the $S_{o}$ mode. The transmitter location was always at the edge of the plate parallel to the notch orientation. The receiver was a wide-frequency type housed within a bubbler mounted in a scanning arm. The receiver signals were sent to a data acquisition system through a pre-amplifier. The scanning table and the acquisition system are controlled using an NDE acquisition software. The signals collected from the receiver as a function of its position were stored for further analysis. Figure 2 illustrates a plate specimen on the scanner system.

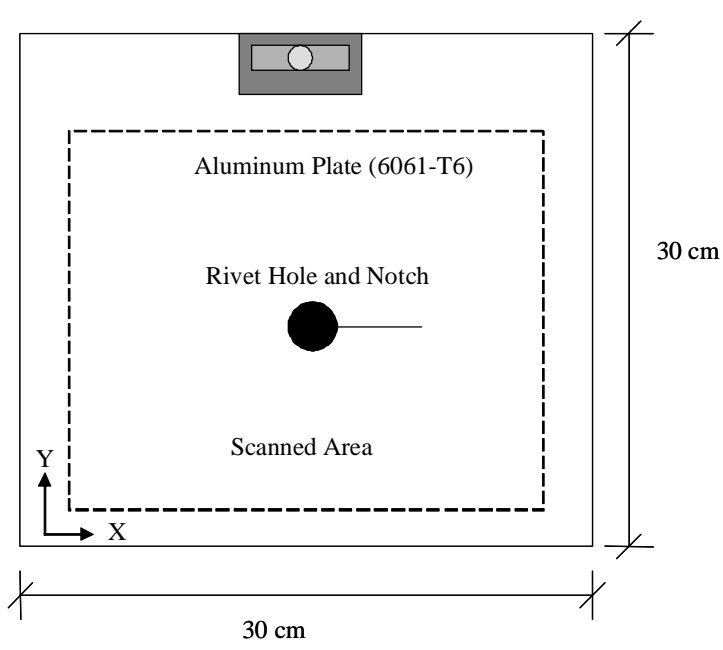

Figure 1: Schematic of Aluminum plate specimens with notches.

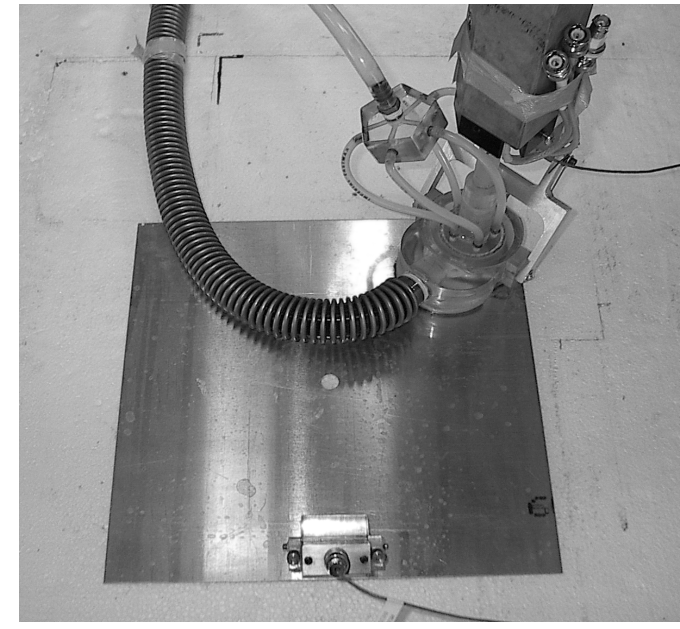

Figure 2: Scanning system with wedge transmitter and receiver within a bubbler. 
In order to select the proper choice of frequency to attain near non-dispersive conditions, experimental phase dispersion curves were obtained and compared to the classical closed form solution originally published by Viktorov [8]. The experimental curves were obtained from data acquired by sending a rectangular pulse to the transmitter while data is collected along a line perpendicular to the transmitter at equally spaced intervals. The double FFT of the data array provides three-dimensional information of the propagating wave amplitudes versus frequency and wave number. Through the use of standard expressions, the graph is transformed to show the propagating wave amplitudes versus frequency and phase velocity to obtain the experimental phase dispersion curve. This curve is illustrated in Figure 3 along with Viktorov's [8] analytical solution using the geometric and materials properties corresponding to the plate specimens. A good correlation was obtained. This experiment was also helpful to evaluate the frequency limitations of the transmitter and the receiver system. Reasonably good information was obtained for frequencies less than $2 \mathrm{MHz}$. In order to avoid different group and phase velocities, further tests were conducted by sending a $500-\mathrm{kHz}$ tone burst. The $\mathrm{S}_{\mathrm{o}}$ mode propagates at a phase velocity of $5,280 \mathrm{~m} / \mathrm{s}$ at this frequency.

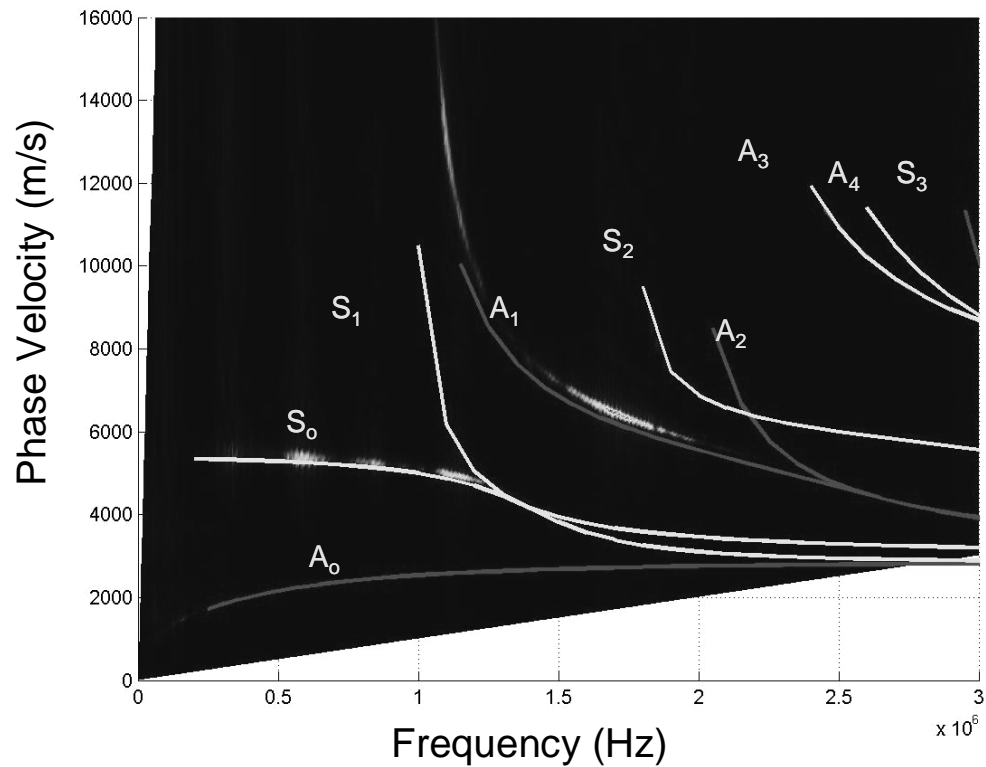

Figure 3: Experimental phase dispersion contour plot vs. analytical solution for an Aluminum plate $1.6 \mathrm{~mm}$ thick.

In order to establish a scanning strategy, it is necessary to visualize the Lamb-wave scattering and reflections from the combined hole and notch. Scans were conducted over an area of $150 \mathrm{~mm}$ by $150 \mathrm{~mm}$, as illustrated in Figure1. The time histories were stored. Pixel-valued images were created by color coding the values of all measurement points at the same time reference as a function of the measurement position. The series of images were organized in sequence to generate an animation displaying the propagation of the waves. Figure 4 illustrates the amplitude of the waves at four different times. The first one illustrates the wave front prior to reaching a hole with a $6.4-\mathrm{mm}$ notch. The second one illustrates the wave front meeting the hole. The third one shows the image at a time where the waves are beginning to be scattered by the hole and reflected from the notch. The last image clearly illustrates the transmitted waves and the combined scattered and reflected waves. These animations served as a visualization tool; however because of the number of waves involved in the travel group, the visualizations of the notch reflections are overshadowed by the scatter from the hole. For this reason, an alternate approached was followed which involved the animation of the signal energy obtained through the Hilbert-Huang transform [9] 


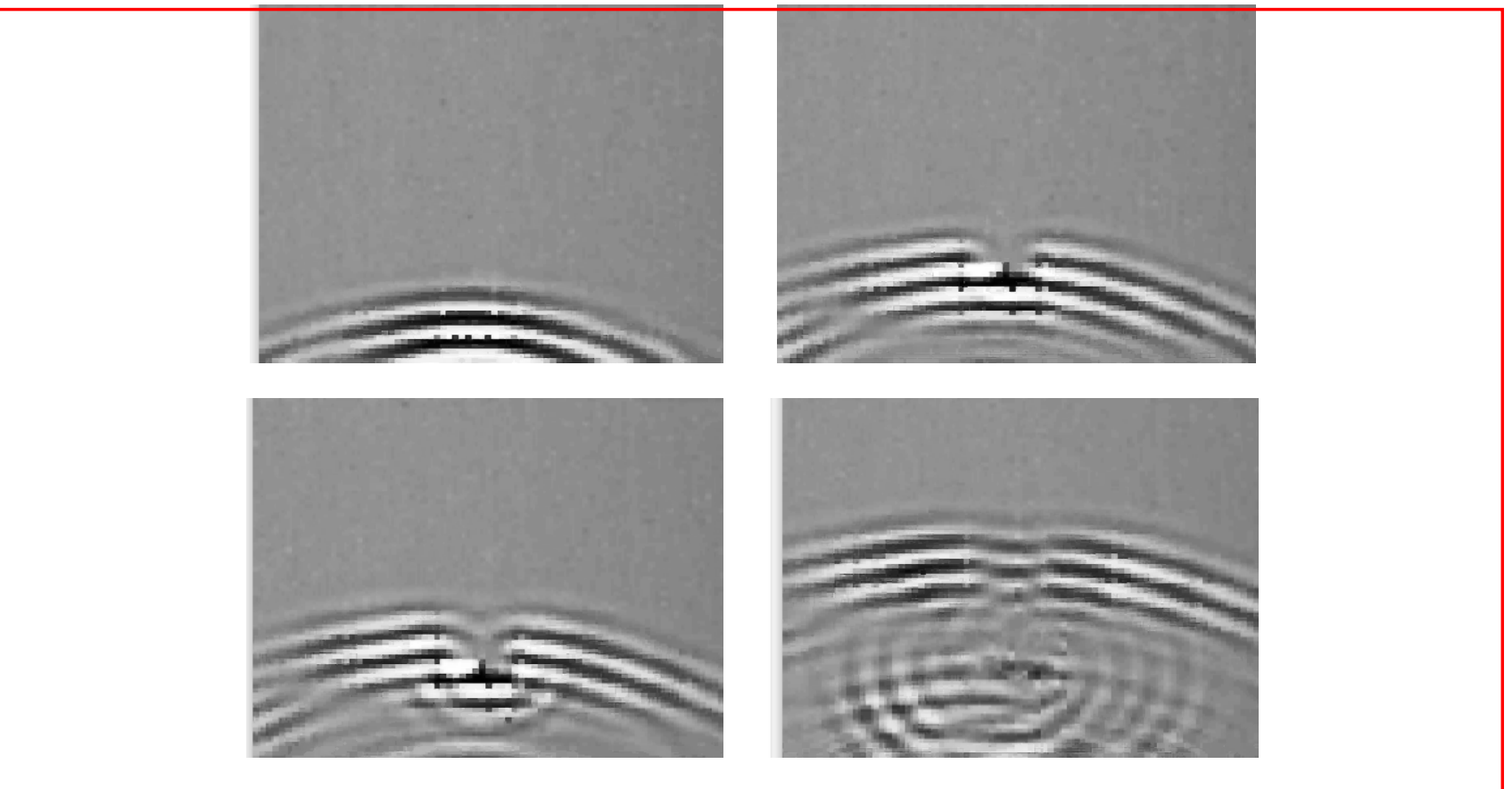

Figure 4. Visualization of propagating $\mathrm{S}_{\mathrm{o}}$ Lamb wave before and after reaching a rivet hole with a 6.4-mm notch.

\section{DETERMINATION OF SIGNAL ENERGY}

The Hilbert-Huang Transform is a recent breakthrough in the analysis of nonlinear nonstationary time series analysis [9] which allows for the separation of the time series into intrinsic mode functions and a residue. The separation consists of a sifting process obtained by detecting the maximum and minimum peaks of the time series, computing of the maximum and minimum envelopes through a curve fitting approach, and subtracting the signal from the mean of the envelopes. For further details of the sifting process and the determination of the intrinsic mode functions see reference [9]. At the end, the intrinsic mode functions have the properties that the numbers of extrema and zero crossings must be equal or differ by one, and that at any point in time, the mean value of the envelopes defined by the local maxima and local minima is zero [9]. Figure 5 illustrates the results of the sifting process applied to a typical ultrasonic signal acquired by the receiver. The first graph is the original signal. The rest of the time series are the intrinsic mode functions, $C_{k}$, as obtained by applying the algorithms described in [9]. The last time series corresponds to the residue. When the $C_{k}$ 's are added, the original signal is recovered. It should be noted that the effect of the sifting, in context to Lamb waves, is filtering the data in a band-pass form. In essence, the time series is separated by frequency ranges, whose wave groups may travel at different velocities. For example, the experiment phase dispersion curves associated with the first intrinsic functions of an array of time series acquired by sending a $500-\mathrm{kHz}$ tone burst to the transmitter and collecting data at equally spaced intervals along a line in the direction of the wave propagation is illustrated in Figure 6 . Noise and the coincidental generation of waves at different frequencies can be controlled limiting the number of intrinsic mode functions.

Once the mode separation is complete, the Hilbert transform is applied to each intrinsic mode function, $C_{k}$, to obtain:

$Y_{k}(t)=\frac{1}{\pi} P \int_{-\infty}^{\infty} \frac{C_{k}\left(t^{\prime}\right)}{t-t^{\prime}} d t^{\prime}$ 

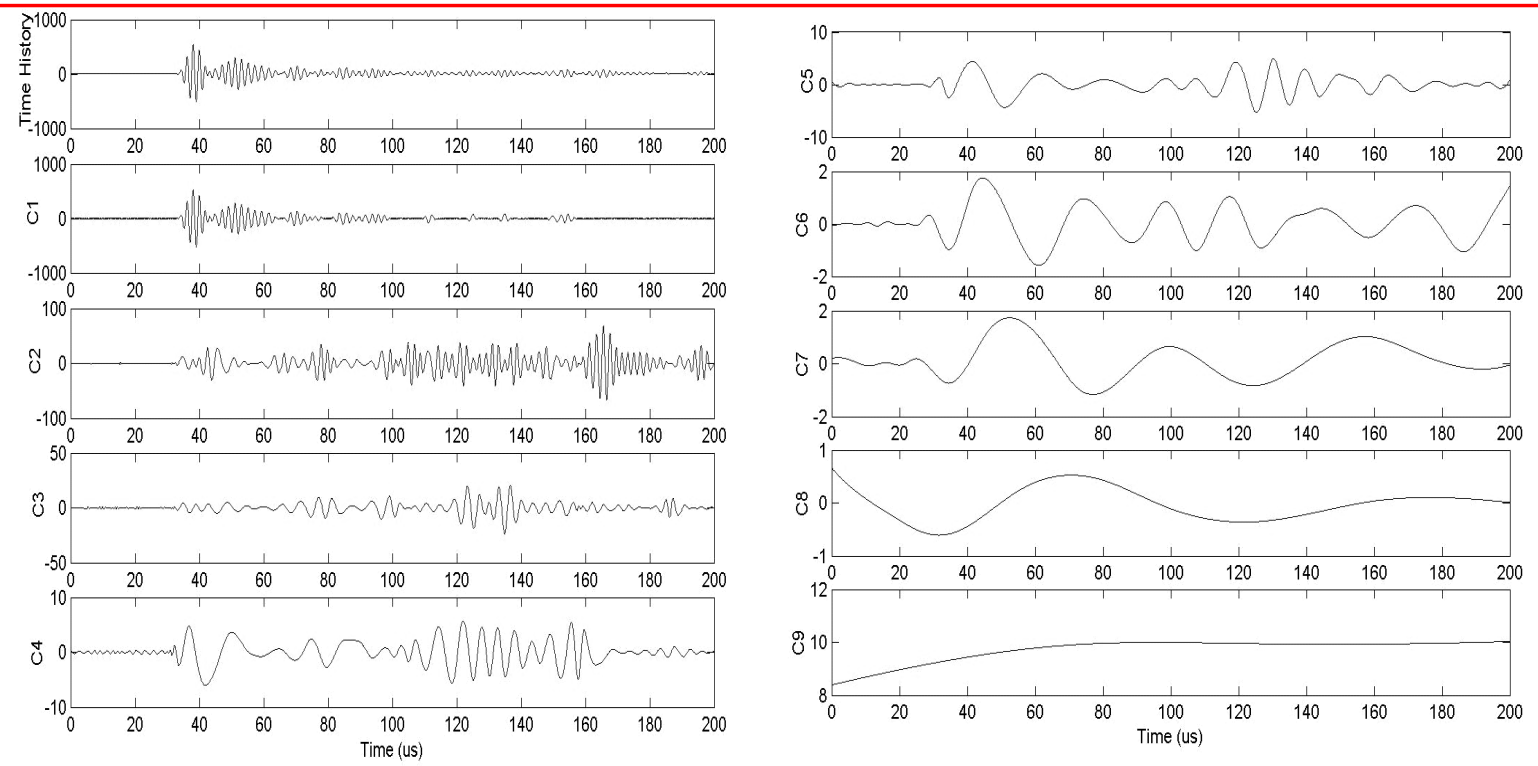

Figure 5. Intrinsic mode functions by applying the sifting process to a typical receiver signal.

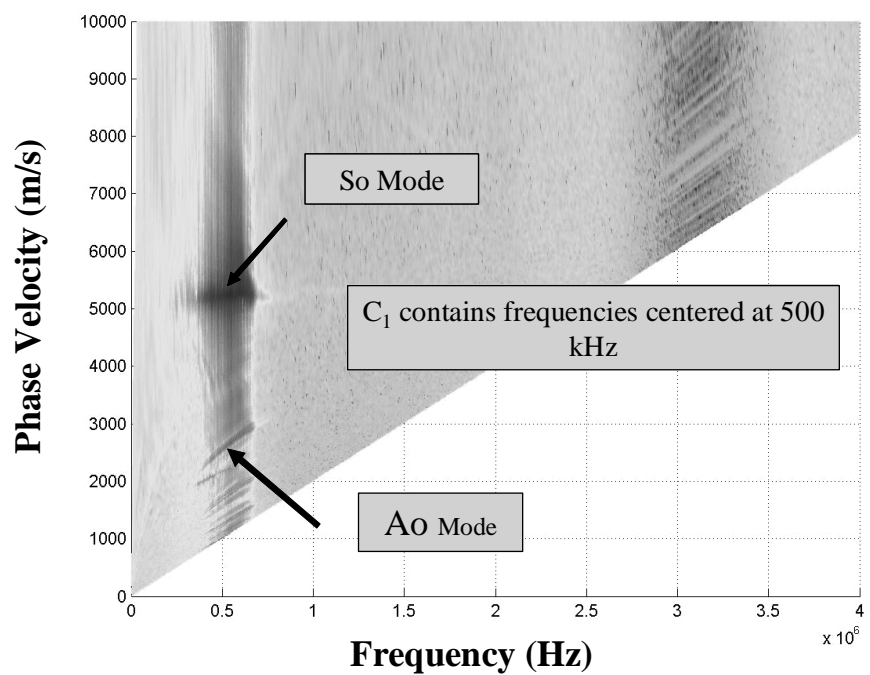

Figure 6: Experimental phase dispersion contour plot of Intrinsic Mode Function $\mathrm{C}_{1}$, for an Aluminum plate $1.6 \mathrm{~mm}$ thick.

Where $Y_{k}(t)$ is the Hilbert transform of $C_{k}$. Then, the analytical signal is formed by retaining the original signal in the real part and the Hilbert transform in the imaginary part, to give:

$Z_{k}(t)=C_{k}(t)+i Y_{k}(t)=a_{k}(t) e^{i \theta t}$

$a_{k}(t)=\left|Z_{k}(t)\right|$

Where $a_{k}(\mathrm{t})$ is the amplitude of the analytical signal. 
$E_{k}(t)=\frac{1}{2} a_{k}^{2}(t)$

The total signal energy as a function of time energy at one point is the superposition of the energies computed for each intrinsic mode function, to give:

$E(t)=\sum_{k=1}^{n} E_{k}(t)$

Where, $\mathrm{n}$ is the number of sifts to be considered. For the experiments described here, a total of three intrinsic mode functions were needed to focus the analysis in the frequency band centered at $500 \mathrm{kHz}$.

The time histories for the area scans were processed to compute the signal energy of the first three intrinsic mode functions as described in equations (1) through (5). Animations were similarly created color coding the energy values of all measurement points at the same time reference and sequencing the images in time. This allowed for animations displaying the propagation of the waves' energy. Figure 7 illustrates the amplitude of the wave energy at four different times in a similar fashion as Figure 4. It should be observed that the presence of the notch is clearly visible in the second and third images. Furthermore, the last image clearly illustrates the differences between the energy backscattered from the hole and that reflected from the notch, as well as the effects of the Lamb wave passed through the hole and the notch. Therefore, it seems that two possible strategies are feasible to characterize potential notches: (a) scanning along a line between the transmitter and the hole to analyze the arrival times and amplitudes of refractions and reflections, and (b) scanning along a line passed the rivet hole. This paper only addresses the first option.
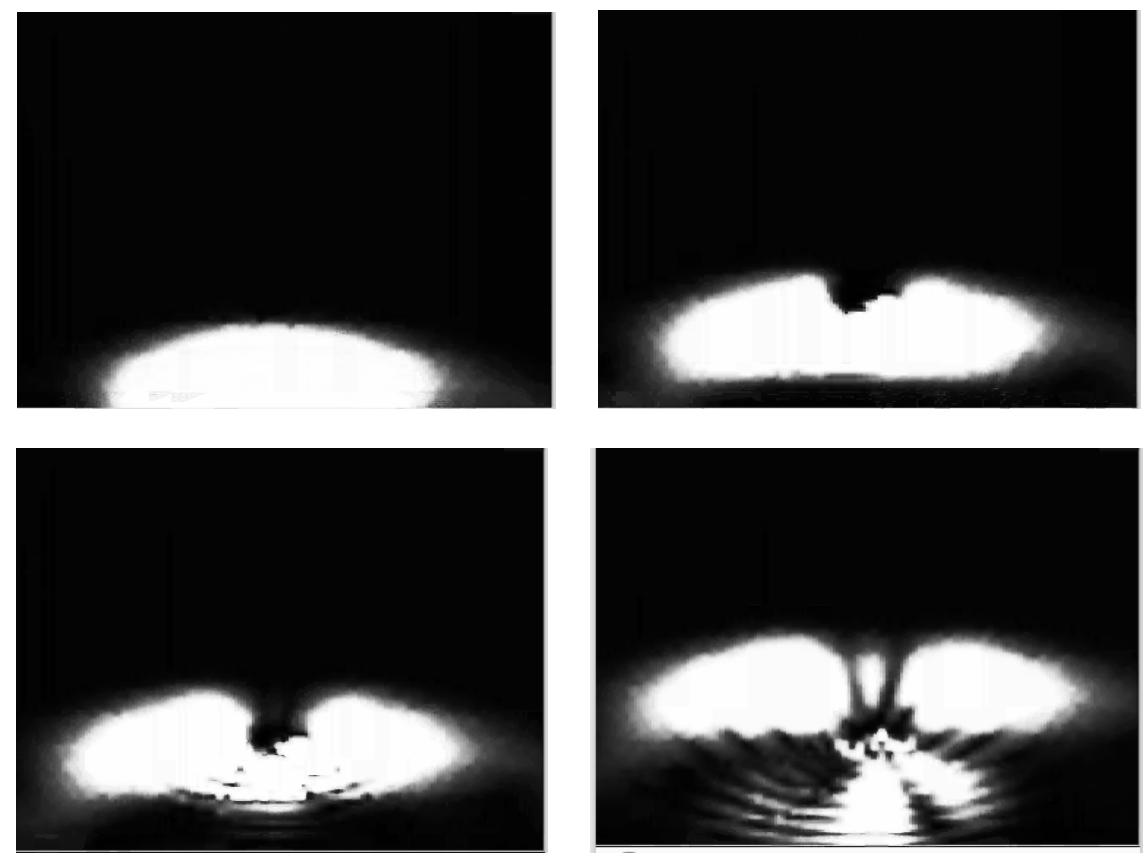

Figure 7. Visualization of propagating $S_{o}$ Lamb wave energy before and after reaching a rivet hole with a 6.4-mm notch. 


\section{LINE SCANNING APPROACH}

In order to implement a solution for the notch characteristics at the rivet hole, receiving signals are required along a line that can pick up notch-reflected wave energy. Knowing the arrival times of notch-reflected waves, it is possible to use the information of two or more receiver locations to calculate the location of points on the surface that cause those reflections. However, the data measured along a line additionally picks up the scattered waves from the hole geometry. These would need to be separated. This paper considers a scanning approach along a line that is parallel to the notch orientation, as depicted in Figure 8, where the signals from the receivers at the known positions can be processed to determine arrival times and coordinates of the source of reflections, providing information about the notch geometry. The transmitter was placed at the edge and placed so that the ultrasonic pulse is directed towards the rivet hole. The scanned line was parallel to the edge at a distance of $120 \mathrm{~mm}$. A total length of $150 \mathrm{~mm}$ was scanned and measurements taken at $1 \mathrm{~mm}$ intervals. Such a scanning approach has the potential to be implemented in real-time.

All the acquired time series were processed using the Hilbert-Huang transform and the information was converted to signal energy using equations (1) through (5) and using the first three intrinsic mode functions. Figure 9 (a) illustrates the signal wave energy as functions of time for all points along the scanned line. The horizontal axis corresponds to the time interval number, and the $y$ axis corresponds to artificial offsets to visualize all the points along the line. The figure illustrates bumps corresponding to wave energy at different times. The first major bump corresponds to the front wave arriving at the receiver locations. The second major bumps correspond to a wave energy combination of scattered waves from the hole and reflected energy from the notch. The rest of the waves correspond to secondary reflections from the edges. It should be noted that the trigger was synchronized with the pulse leaving the function generator. There is a time delay corresponding to $21.1 \mu s$ (132 time steps) or the time it takes the wave to leave the Plexiglas wedge into the Aluminum plate after the trigger. The peaks of the energy provide the information of the arrival times along wave pathways, and since the receiver and the transmitter positions and the propagation velocity are known, distances along the pathways can be determined. Figure 9 (b) illustrates the arrival times of energy peaks that are above a given threshold. Figure 10 illustrates the maximum amplitude of the wave energy scattered by the hole versus the receiver position, corresponding to a control plate with the hole without a notch. This graphs illustrates that the arrival times due to hole back scattering occur from 50 to $90 \mathrm{~mm}$ along the scan line. Reflections due to the notches would occur outside this region.

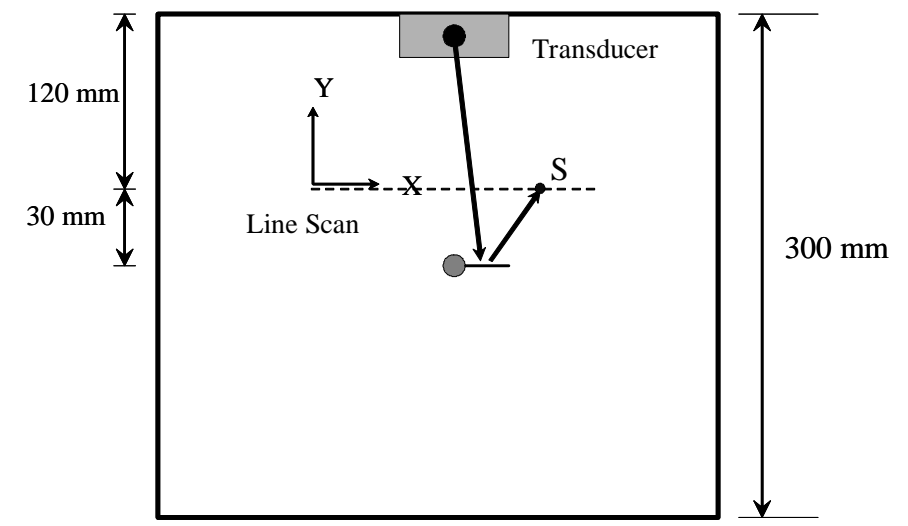

Figure 8: Schematic of line-scan approach to characterize notch characteristics. 


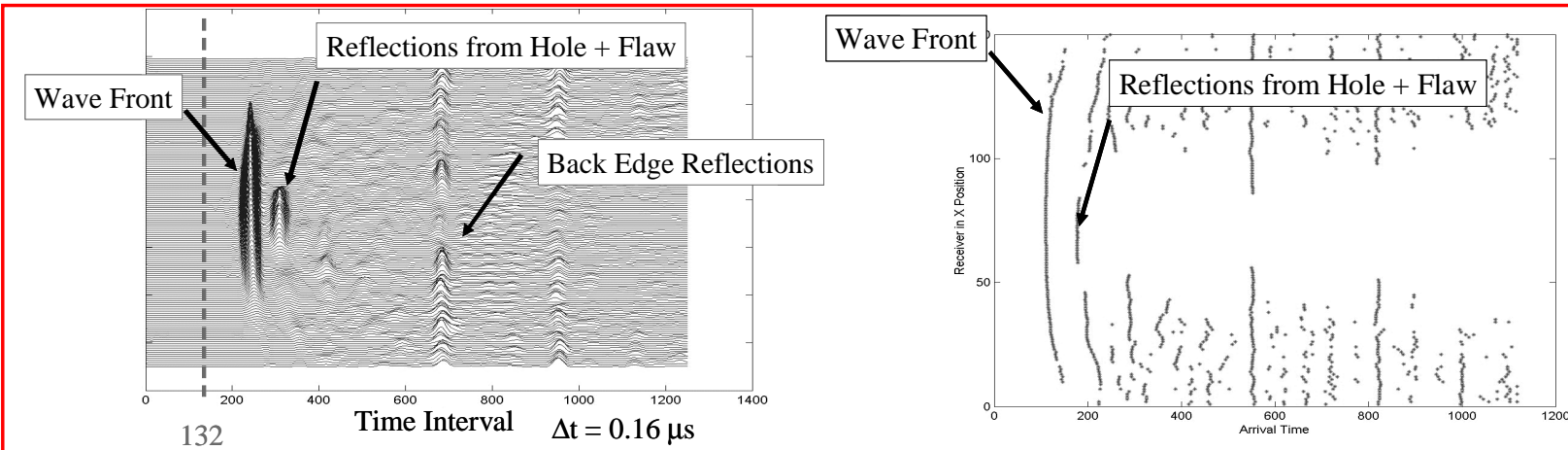

Figure 9: (a) Display of wave energy along points of a typical line scan. (b) Arrival times of energy pulse.

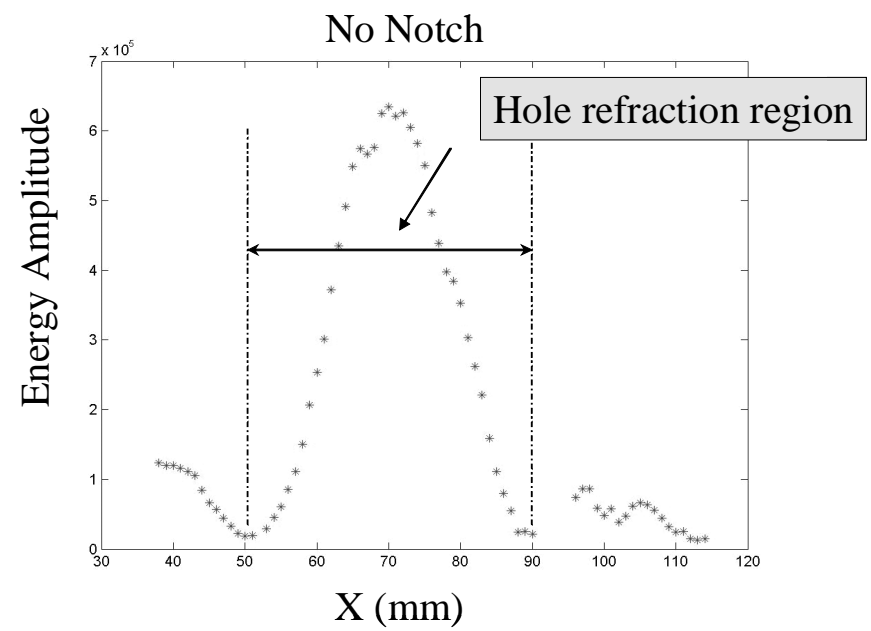

Figure 10: Amplitude of scattered wave energy from hole without notch along line of scan.

\section{SOLUTION OF NOTCH LOCATION}

Coordinates of points on a notch that causes reflections can be obtained using the times of flight of the incident and reflected waves from a fixed transmitter to a receiver at two different points. The approach is documented in reference [10] and considers a fixed transmitter with a fixed focal source at $F$ and two receivers at locations $F_{1}$ and $F_{2}$ as shown in Figure 11. All focal coordinates are known. The transmitter configuration is as such as to cause Lamb wave excitations of a mode in the non-dispersive region with waves propagating radially at a constant phase velocity. The signals received at $F_{1}$ and $F_{2}$ would consist of the incident wave front, followed by the flaw-reflected waves. From the arrival times of the wave packets and since the waves propagate at a near constant velocity, then the distances $\overline{F E_{1} F_{1}}$ and $\overline{F E_{2} F_{2}}$ can also be found. If these reflections come from the same flaw (points $E_{1}$ and $E_{2}$ ), then the distances $\overline{F E_{1} F_{1}}$ and $\overline{F E_{2} F_{2}}$ are constants along two ellipses with foci at $F$ and $F_{l}$ and $F$ and $F_{2}$, respectively. The main problem is to determine the coordinates of points $E_{l}$ and $E_{2}$ that form a tangent line to both ellipses. The solution is documented in [10] and is not repeated here. 


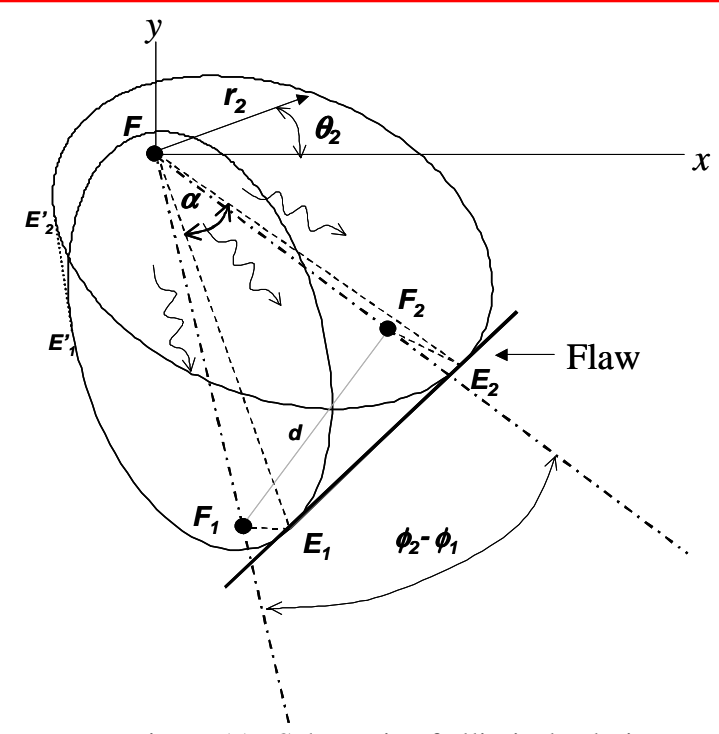

Figure 11: Schematic of elliptical solution.

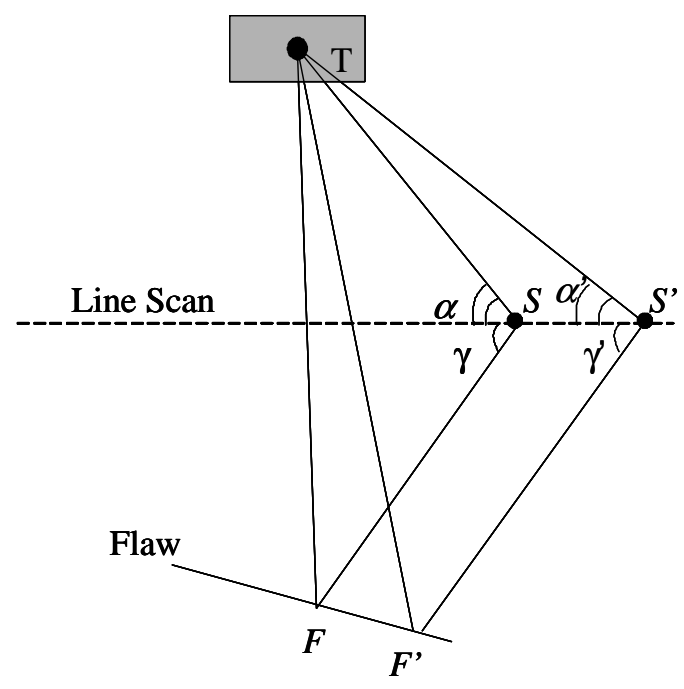

Figure 12: Schematic of geometric solution.

An alternate approach considers the solution formulated in context to Figure 12, where the line of the scan is assumed perpendicular to the line of directed energy of the transmitter. This figure considers the pathways from a fixed transmitter $(T)$ to the receivers at two different points along the line scan $\left(S\right.$ and $\left.S^{\prime}\right)$. The wave paths to the first receiver position are TS and TFS; those for second receiver position are $T S^{\prime}$ and $T F{ }^{\prime} S^{\prime}$. Notice that distances of $T S$ and $T S$ ' are known from the coordinates of the transmitter and the receiver positions. Also note that the distances of the paths TFS and $T F^{\prime} S^{\prime}$ are computed from the arrival times and the wave propagation velocity. The angles $\alpha$ and $\alpha$ can be determined from the geometry. If the distance between the two receiver positions is assumed small compared to the distances along the travel paths, then the angles $\gamma$ and $\gamma$ can be assumed the same. Then, from the geometry it can be proven that the angle $\gamma$ can be approximated through the expression:

$\cos \gamma=\frac{\overline{T F^{\prime} S^{\prime}}-\overline{T F S}}{\overline{S S^{\prime}}}$

Where the bar notation indicates the distances between the referenced points in Figure 12.

The distances of the paths $F S$ and $F^{\prime} S^{\prime}$ can be found through implementation of the Law of Cosines using triangles $T F S$ and $T F^{\prime} S^{\prime}$ with angles $(\alpha+\gamma)$ and $\left(\alpha^{\prime}+\gamma\right)$, respectively. This approach results in the following expression:

$\overline{F S}=\frac{\overline{T F S}^{2}-\overline{T S}^{2}}{2(\overline{T F S}-\overline{T S} \cos (\gamma+\alpha))}$

A similar equation exists for the second triangle using the prime notation.

Once this information is computed, the coordinates of points $F$ and $F^{\prime}$ are readily determined from the geometry, to resolve for the coordinates at points on the notch.

As pointed out in Section 4, the arrival times due to the hole back scattering occur from 50 to $90 \mathrm{~mm}$ along the scan line. Figure 13 illustrates the amplitude of the waves scattered by the hole combined with reflections from notches of different sizes, versus the receiver position. It should be observed that the location of the maximum value shifts to the right as the notch size increases. The figure also illustrates the receiver locations that were used to implement the 


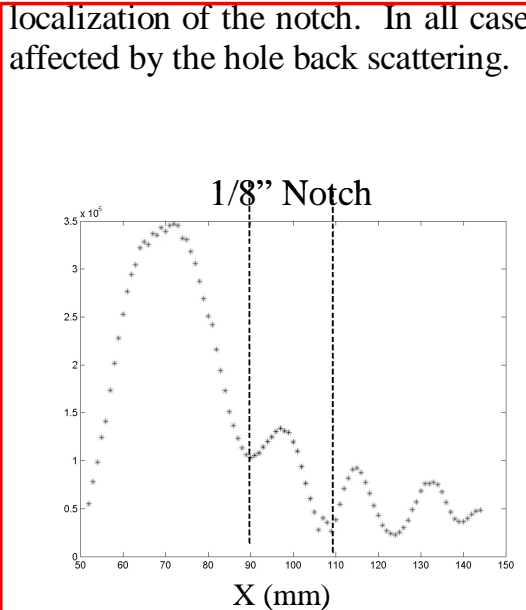

(a) $1 / 8$ " Notch

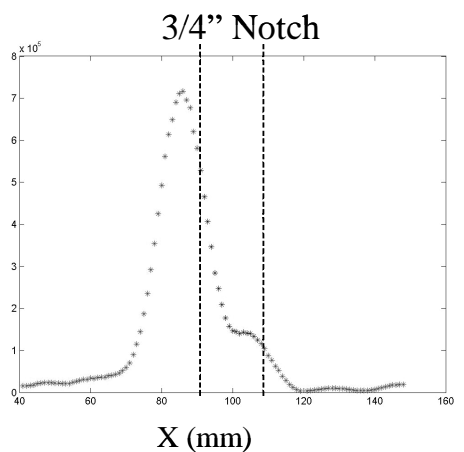

(d) 3/4" Notch

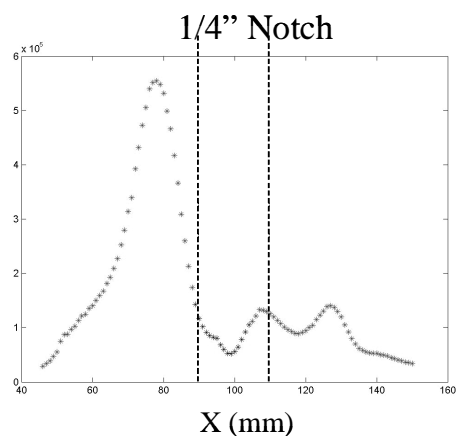

(b) $1 / 4 "$ Notch

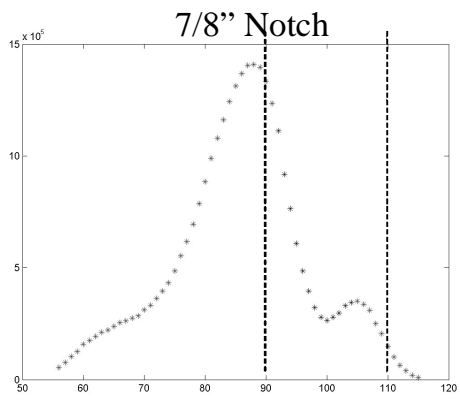

$\mathrm{X}(\mathrm{mm})$

(e) $7 / 8 "$ Notch

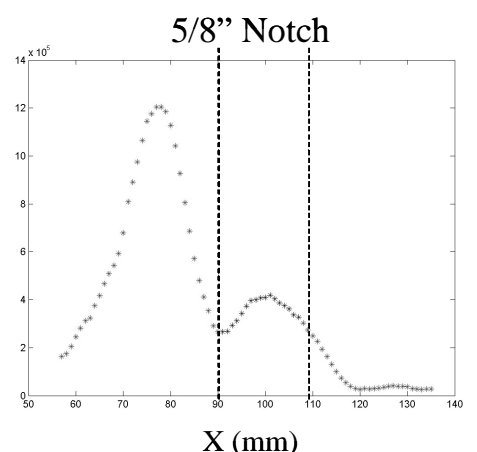

(c) $5 / 8 "$ Notch

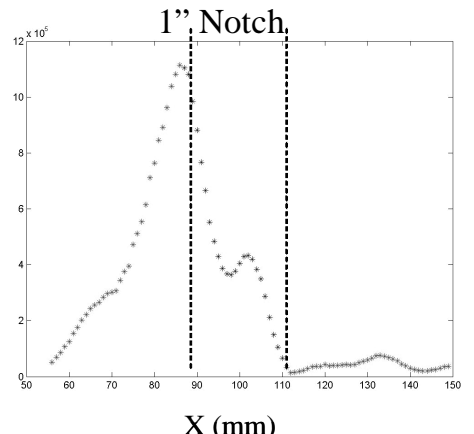

(f) 1 " Notch

Figure 13. Amplitude of scattered and reflected wave energy from hole with a notch along line of scan.

\section{LOCALIZATION RESULTS}

Figure 14 illustrates the results of processing the receiver signals acquired along the line for the plates with a center hole with notches ranging from $3.2 \mathrm{~mm}$ to $25.4 \mathrm{~mm}$ notch as illustrated in Figure 8 . The acquired signals were processed with the Hilbert-Huang transform to determine the signal energy as indicated in Section 3. After correcting for the time delay associated with the trigger and wave arrival into the plate, the times of flight and the amplitudes of the scattering and the reflected waves from the hole and the notch were obtained (Figure 12). The geometric approach as described in Section 6 was implemented for the times of flight corresponding to the range of 90 to $110 \mathrm{~mm}$ as illustrated in Figure 13. The results in Figure 14 include a drawing of the actual hole and notch and crosses indicating the points where the notch localization approach determined a source of reflection. Figure 14 in essence illustrates the location of the predicted notch compared to the actual notch. From the figure, it is observed that the orientation of the notch is accurately predicted. The extent of the flaw was also predicted with excellent agreement for all notch sizes, except for the $3.2 \mathrm{~mm}$ notch, in which the extend of the predicted flaw was overpredicted. 


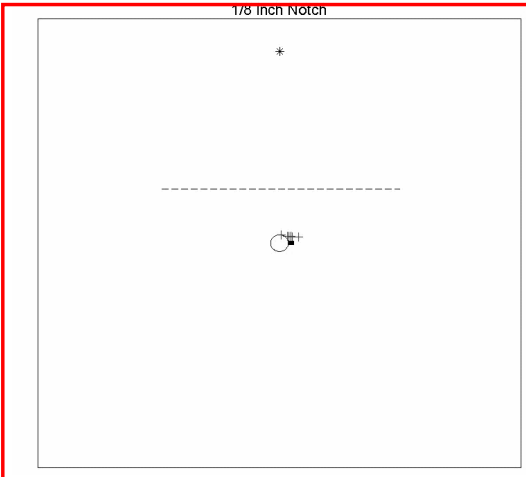

(a) $1 / 8$ " Notch

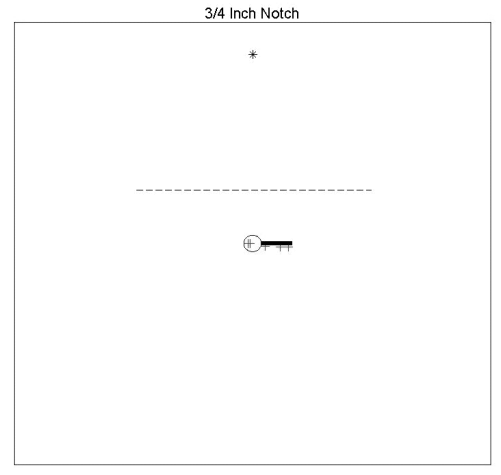

(d) 3/4" Notch

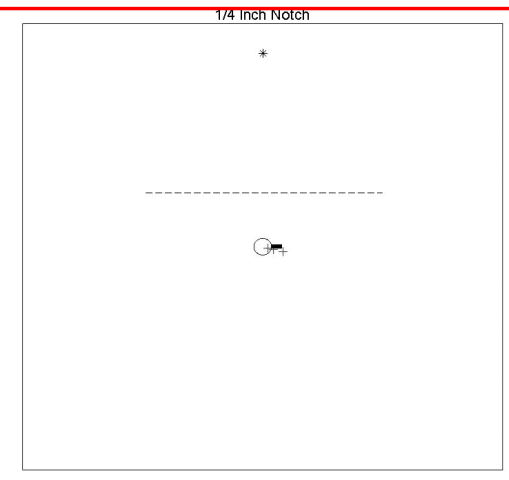

(b) $1 / 4 "$ Notch

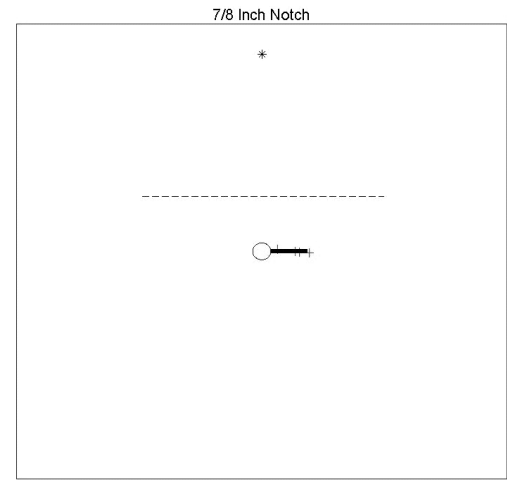

(e) 7/8" Notch

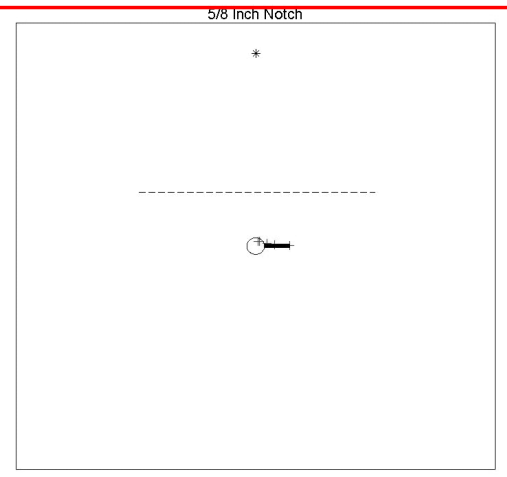

(c) 5/8" Notch

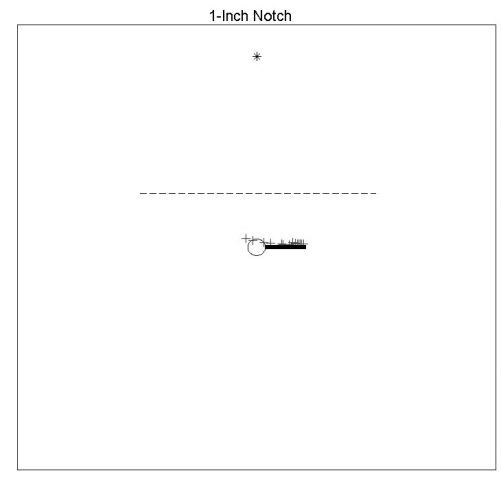

(f) 1 "Notch

Figure 14: Notch localization results using the geometric approach compared to the actual notch.

\section{CONCLUSIONS}

This paper has described a Lamb-wave scanning method for the detection of notches simulating cracks at rivet holes in thin plates. The approach requires the generation of an ultrasonic $S_{0}$-Mode Lamb wave with a tone burst centered at a near non-dispersive frequency. Area scans were performed of a plate with a hole with a notch to generate times series information which was used to create animations to visualize the propagation characteristics. The time series were subjected to a sifting process to obtain intrinsic mode functions. The Hilbert-Huang transform was applied to the intrinsic mode functions which permitted the computation of the signal energy as a function of time. Animations of the propagation of the Lamb-wave energy clearly illustrated that a scanning approach consists of acquiring time series along a line between the transmitter and the hole to capture wave scattering from the hole and reflections from the notches. The times of flight and amplitudes of the notch-reflected energy outside a region affected by the back scattering from the holes were used to calculate coordinates of the source of the reflections by a geometric approach. Line scans were conducted on various square thin Aluminum plates with a center hole and various full-thickness notches ranging from $3.2 \mathrm{~mm}$ to $25.4 \mathrm{~mm}$ long. The notches were perpendicular to one of the sides

The series of identified coordinates outlines the extent of the notch at the rivet hole. Results of experiments conducted on thin square plates with a single hole with notches of various sizes compare favorably with the actual notches.

The following conclusions are stated on the proposed scanning approach: 
- The location of the notch was always detected and correctly outlined.

- The extent of the flaw was accurately predicted for all the notches, except for the $3.2 \mathrm{~mm}$ long notch case.

- The extent of the flaw was over-predicted for the shortest notch.

- The orientation of the predicted flaw was always in agreement with the actual notch orientations.

\section{ACKNOWLEDGEMENTS}

Efforts sponsored by the Air Force Office of Scientific Research, Air Force Material Command, USAF, under grant number F49620-00-1-0365. The US Government is authorized to reproduce and distribute reprints for governmental purposes notwithstanding any copyright notation thereon. This paper is based on the master's thesis of Mr. Enrique Roldan.

\section{REFERENCES}

1. P. Cawley, D. Alleyne, "The use of Lamb waves for the long range inspection of large structures," Ultrasonics 34, 1996, 287-290.

2. T. Kundu, K. Maslov, P. Karpur, T.E. Matikas, P.D. Nicolau, "A lamb wave scanning approach for the mapping of defects in [0/90] titanium composites," Ultrasonics 34, 1996, 43-49.

3. M.J.S Lowe, D.N. Alleyne, and P. Cawley, "Defect detection in pipes using guided waves," Ultrasonics 36, 1998, $147-154$.

4. T. Ghosh, T. Kundu and P. Karpur, "Efficient use of Lamb modes for detecting defects in large plates," Ultrasonics 36, 1998, 791-801.

5. T. Kundu, A. Maji, T. Gosh and K. Maslov, "Detection of kissing bonds by Lamb waves," Ultrasonics 35, 1998, 573-580.

6. W. Zhu, J.L. Rose, J.N. Barshinger, V.S. Agrawala, "Ultrasonic guided wave NDT for hidden corrosion detection," Res. Nondestr. Eval. (1998), 10, 205-225.

7. Z. Chang and A. Mal, "Scattering of Lamb waves from a rivet hole with edge cracks," Mechanics of Materials, 31, 1999, 197-204.

8. I.A. Viktorov, Rayleigh and Lamb Waves - Physical Theory and Applications, Plenum Press, New York, 1967.

9. N.E. Huang, Z. Shen, S.R. Long, S.R., M.C. Wu and Shih, H. H., et al., "The Empirical Mode Decomposition and Hilbert Spectrum for Nonlinear and Nonstationary Time Series Analysis,". Proc. Royal Society of London Series, A454, 1998, 903-995.

10. F. DeVilla, E. Roldan, C. Tirado, R. Mares, S. Nazarian and R. Osegueda, "Defect Detection in thin plates using So Lamb wave scanning," in Proc. of NDE for Health Monitoring and Diagnostics, SPIE Vol 4335, Paper 21, March 2001. 